Revue d'histoire de l'Amérique française

\#IS REVUE D.HISTOIRE DE L'AMÉRIQUE FRANÇAISE

\title{
Tendances dominantes de l'historiographie religieuse au Canada anglais, 1979-1988
}

\section{Paul Laverdure}

Volume 42, numéro 4, printemps 1989

URI : https://id.erudit.org/iderudit/304739ar

DOI : https://doi.org/10.7202/304739ar

Aller au sommaire du numéro

Éditeur(s)

Institut d'histoire de l'Amérique française

ISSN

0035-2357 (imprimé)

1492-1383 (numérique)

Découvrir la revue

Citer cette note

Laverdure, P. (1989). Tendances dominantes de l'historiographie religieuse au Canada anglais, 1979-1988. Revue d'histoire de l'Amérique française, 42(4),

579-587. https://doi.org/10.7202/304739ar d'utilisation que vous pouvez consulter en ligne.

https://apropos.erudit.org/fr/usagers/politique-dutilisation/ 


\title{
TENDANCES DOMINANTES DE L'HISTORIOGRAPHIE RELIGIEUSE AU CANADA ANGLAIS, 1979-1988 ${ }^{1}$
}

\author{
PAUL LAVERDURE \\ Département d' histoire \\ Université de Toronto
}

L'historiographie religieuse anglophone des dix dernières années se présente à première vue comme un pot-pourri incompréhensible. Il existe une telle abondance de publications qu'une division en trois permet de mieux s'y retrouver ${ }^{2}$. D'autres historiens pourraient établir des catégories différentes et nos divisions ne sont pas étanches, mais les nouveaux venus y trouveront d'utiles points de repère révélateurs des tendances dominantes: la première pratique l'histoire théologique, la seconde, une histoire politique du fait religieux, et la troisième une histoire religieuse et sociale de l'Église, importée surtout de Chicago. Par le premier courant, nous voulons identifier l'histoire théologique d'allure apologétique; elle privilégie le genre biographique. Le second courant, surtout pratiqué par les historiens de Toronto, fait de l'histoire politico-religieuse en cherchant à situer le christianisme en tant que religion dominante dans le contexte de l'évolution «nationale» du pays. Le troisième courant s'inspire de la rencontre des sciences sociales et de l'analyse comparative. Si chaque courant suit sa voie sans trop se soucier des autres, il semble que les nouveaux historiens privilégient l'histoire sociale aux dépens de l'histoire politique.

Avant la Deuxième Guerre mondiale, tous les historiens du christianisme, anglais et français, se réjouissaient de faire, tel Eusèbe, l'éloge des fondateurs et de leurs partisans. Leurs livres essayaient de montrer le dessein divin dans l'histoire d'une âme, des hommes (de peu de femmes), ou d'une Église paroissiale, nationale ou mondiale, de confession anglicane, baptiste, réformée, etc. Dans ces histoires, il s'agis-

1 Je tiens à remercier Serge Gagnon qui a revu une première version de cette note critique. Ce texte a d'abord fait l'objet d'une communication au Congrès de l'Institut d'histoire de l'Amérique française, le 22 octobre 1988 , à Trois-Rivières.

2 Michel Despland a adopté une division semblable dans «Editorial. Church History, History of Christianity, and History of Religions», Sciences religieuses, 7,3 (1978): 259-261. Voir aussi Louis Rousseau, «La triple appartenance de l'histoire religieuse actuelle», Stryckman et Rouleau, Sciences sociales et Eglises. Questions sur l'évolution religieuse du Québec (Montréal, Bellarmin, 1980), 327-331. 
sait de réécrire la Bible, la biographie d'un dieu-fait-homme et l'histoire du salut d'un peuple. Avant 1960, au Canada anglais, dans les collèges axés sur la formation de pasteurs, d'avocats et de médecins, les historiens de l'Église étaient des théologiens pour qui l'histoire, comme toute autre discipline, était au service de la théologie et de la pastorale.

Parmi les historiens du courant «théologique», John Webster Grant travaille pour l'Église unie, la plus grande confession protestante au Canada. Anciennement professeur au Collège Emmanuel de l'Université de Toronto, auteur et ardent défenseur de l'oecuménisme, Grant incarne la théologie libérale protestante dans ses oeuvres historiques sur l'Église unie. Son livre, Moon of Wintertime, a été accueilli par certains comme un ouvrage inspirant et inspiré. Mais les historiens ont manifesté des opinions divergentes à propos de cet essai qui cherche à comprendre les rapports entre les autochtones et les missionnaires. Un critique, par exemple, a accusé Grant d'écrire en théologien plutôt qu'en historien sur les croyances actuelles des Églises devant l'ancien problème d'indigénisation de la foi ${ }^{3}$. Au sein des catholiques, anglophones comme francophones, la venue du pape Jean Paul II a créé une véritable industrie de "piété historique» chez les théologiens ${ }^{4}$. La multiplication des ouvrages indique à quel point les ressources sont disponibles pour les productions hagiographiques ${ }^{5}$. Les biographies d'évêques, de prêtres et de religieuses prédominent. Du côté anglican, cette tendance compte aussi des adeptes. Le meilleur écrivain anglican est le révérend Thomas Millman, maintenant retraité, tout comme John Webster Grant ${ }^{6}$. Parmi les baptistes, les recueils de Jarold Zeman sont importants ${ }^{7}$. Tant chez les baptistes que chez les presbytériens, l'historiographie est en

3 John Webster Grant, Moon of Wintertime: Missionaries and the Indians of Canada in Encounter Since 1534 (Toronto, University of Toronto Press, 1984). Les comptes rendus de John Badertscher, Sciences religieuses, 14,1 (1985): 109-111, et de Olive Patricia Dickason, Canadian Historical Review, 66,2 (1985): 267, énoncent deux appréciations divergentes de l'ouvrage.

4 Pour les francophones, voir Guy LaPerrière, "Centenaires de diocèses et histoire régionale au Canada français», La Reviue d' histoire ecclésiastique, 82,1 (1987): 44-50.

5 Cyril J. Byrne, ed., Gentlemen-Bishops and Faction Fighters: the Letters of Bishops O'Donel, Lambert, Scallan and Other Irish Missionaries (St.John's [Terre-Neuve], Jesperson Press, 1984). Paul O'Neil, Upon this Rock. The Story of the Roman Catholic Church in Newfoundland and Labrador (St.John's [Terre-Neuve], Breakwater Books, 1984). J. Brian Hanington, Every Popish Person. The Story of Roman Catholicism in Nova Scotia and the Church of Halifax, 1604-1984 (Halifax, Archidiocese of Halifax, 1984). Marie-Louise Brugeyroux, Friars and Friends. A History of St.Francis of Assisi Parish 1909-1984 (Edmonton, St.Francis of Assisi Parish, 1984).

6 Thomas R. Millman, Atlantic Canada to 1900: a History of the Anglican Church in Atlantic Canada (Toronto, Anglican Book Centre, 1983).

7 Par exemple, Walter Ellis, «Baptists and Radical Politics in Western Canada, 1920-1950», Jarold K. Zeman, ed., Baptists in Canada. Search for Identity Amidst Diversity (Burlington [Ont.], G. R. Welch, 1980), 161-182. On peut aussi lire avec profit Murray Ford, Canadian Baptist History and Polity: Papers From the McMaster Divinity College Baptist History Conference (Hamilton, McMaster University Divinity College, 1982). W. Stanford Reid, ed., Called to Witness. Profiles of Canadian Presbyterians. A Supplement to Enduring Witness (Hamilton, The Presbyterian Church in Canada, 1980), volume 2, est un des meilleurs exemples de cette tendance à publier des biographies. Voir aussi les recueils de la Société canadienne d'histoire presbytérienne et de la Société canadienne d'histoire de l'Église. 
plein essor. Ne possédant pas de revue spécialisée comme les autres Églises, ces derniers ont multiplié les monographies et les brochures.

Deux raisons expliquent, selon nous, l'explosion actuelle dans la production d'histoire de l'Église: premièrement, le monde de l'édition est disposé à publier dans tous les domaines; deuxièmement, le monde universitaire, représenté par le presbytérien Moir et le baptiste Rawlyk, est plus apte à en produire. Avec le déclin du nombre de croyants et de théologiens, et à cause des exigences du métier, il est probable que nous verrons encore moins de théologiens se spécialiser en histoire de leur confession respective.

Carl Berger a démontré que l'historiographie canadienne-anglaise de 1900 à 1960 était d'abord l'oeuvre de l'Université de Toronto. Fondé par un pasteur anglican, le département d'histoire de cet établissement n'eut point de querelle avec les théologiens avant la Dépression. Mais, durant les années 1930, la misère, que compensait mal la promesse d'un salut trop lointain, favorisa le développement de l'histoire économique et politique ${ }^{8}$. Un des meilleurs historiens de Toronto, Donald Creighton, biographe de John A. Macdonald, tenta de réunir le courant théologique (grâce à son père, pasteur méthodiste) et celui de l'histoire politique ${ }^{9}$. À l'instigation de Creighton, un de ses étudiants, John Moir, a relevé le défi de vivre comme historien de la religion hors des collèges théologiques ${ }^{10}$. Par ses études des rapports entre les Églises et l'État, Moir est devenu l'un des historiens les plus importants du Canada anglais ${ }^{11}$.

Le deuxième courant, l'école d'histoire politico-religieuse de Toronto, naquit grâce à la collaboration de Moir et du théologien Grant. Il est vite devenu impossible de traiter d'histoire religieuse au Canada anglais sans mentionner ce duo. Leur oeuvre collective, A History of the Christian Church in Canada (1966-1972), est un classique ${ }^{12}$. Entre 1979 et 1988, on a vu Moir et Grant représenter Toronto aux colloques

\footnotetext{
8 The Writing of Canadian History. Aspects of English Canadian Historical Writing Since 1900 (Toronto, University of Toronto Press, 1986), deuxième édition.

9 John Moir, ed., Character and Circumstance: Essays in Honour of Donald Grand Creighton (Toronto, Macmillan, 1970), 1-8.

${ }_{10}$ Comme presbytérien, après 1974, Moir pouvait rejoindre les exigences de la pratique historique avec une foi protestante néo-orthodoxe, c'est-à-dire avec une croyance à la transcendance de Dieu. Moir séparait le sacré et le profane, comme le faisait le dix-neuvième siècle, sa période préférée. Pour les adeptes d'une croyance immanente, unifiante, méthodiste, de l'Église unie, cette distinction était plus difficile.

${ }_{11}$ Church and State in Canada West: Three Studies in the Relation of Denominationalism and Nationalism, 1841-1867 (Toronto, University of Toronto Press, 1959). Church and State in Canada, 1627-1867: Basic Documents (Toronto, McClelland and Stewart, Carleton Series no 3, 1967). The Church in the British Era, From the British Conquest to Confederation (Toronto, McGraw-Hill Ryerson, 1972).

${ }_{12}$ John Webster Grant, ed., A History of the Christian Church in Canada (Toronto, McGrawHill Ryerson): H. H. Walsh, The Church in the French Era, 1, (1966); John Moir, The Church in the British Era, 2, (1972); John W. Grant, The Church in the Canadian Era, 3, (1972). Des trois, Moir était le seul historien professionnel. Le dernier volume, par Grant, vient d'être réédité avec quelques retouches (Burlington, Welch Publishing Company, 1988).
} 
d'histoire des Églises et aux conférences d'histoire presbytérienne. D'ailleurs, ces conférences ont en quelque sorte été fondées par Grant et par Moir ${ }^{13}$. Signalons que ce dernier vient de publier une nouvelle édition de son chef-d'oeuvre, Enduring Witness, sur l'histoire de l'Église presbytérienne au Canada. Le mérite de ce travail réside dans l'équilibre qu'il réussit à maintenir entre l'histoire institutionnelle et biographique et le contexte social et politique. Il a aussi lancé une petite histoire des études bibliques au Canada. Conservant les atouts de Enduring Witness, il a introduit dans A History of Biblical Studies in Canada le contexte intellectuel et académique international, ce qui représente une nouveauté pour les études d'histoire des Églises canadiennes ${ }^{14}$. Moir prend sa retraite en 1989. Pour les synthèses futures de théologie et d'histoire politique, il faudra compter sur d'autres universitaires.

Parmi les baptistes, George Rawlyk travaille sur l'époque britannique des Maritimes, comme Moir l'a fait pour l'Ontario et les presbytériens. Rawlyk et ses collègues universitaires retracent les racines des Églises baptistes dans la révolution et l'histoire américaines ${ }^{15}$. Les illuminés canadiens, soutiennent-ils, étaient les baptistes du XVIIIe siècle.

Les historiens du catholicisme commencent aussi à franchir les bornes biographiques et théologiques en étudiant les questions de haine et de persécution. La section anglaise de la Société canadienne d'histoire de l'Église catholique, comme la Revue canadienne d'histoire, s'intéresse à Louis Riel, à l'Ouest, à l'éducation et à l'immigration irlandaise ${ }^{16}$. Parmi ces études, citons Louis «David» Riel. Prophet of the New World de Thomas Flanagan et Equal Rights: the Jesuits' Estates Act Controversy de J. R. Miller, parues en 1979. La même année, l'oeuvre magistrale de Brian McKillop, A Disciplined Intelligence, décrit

13 Paul Laverdure, «Index. Canadian Society of Church History 1960-1984», Canadian Society of Church History 1985 Papers (1986), 1-53; «The Canadian Society of Presbyterian History. Index of Papers 1975-1985», The Canadian Society of Presbyterian History Papers 1986 (1987), 1-14, permettent de voir leur contribution et celle de leurs étudiants.

John S. Moir, A History of Biblical Studies in Canada. A Sense of Proportion (Chico [California], Scholars Press, 1982). Enduring Witness: a History of the Presbyterian Church in Canada (Toronto, Presbyterian Publications, 1987), deuxième édition. Les anglicans écrivaient un peu l'histoire religieuse sans frontière, mais ils se bornaient aux mouvements missionnaires de l'Angleterre.

is George A. Rawlyk, Ravished by the Spirit: Religious Revivals, Baptists, and Henry Alline (Kingston, McGill-Queen's University Press, 1984). James Beverley and Barry Moody, The Journal of Henry Alline (1982). Gordon T. Stewart, ed., Documents Relating to the Great Awakening in Nova Scotia 1760-1791 (1982). Rawlyk, New Light Letters and Songs (1983). D. G. Bell, ed., Newlight Baptist Journals of James Manning and James Innis (1984). Rawlyk, ed., The Sermons of Henry Alline (1986). Ces derniers sont de Hantsport, N.-É., Lancelot Press, Baptist Heritage in Atlantic Canada Series. Rawlyk a aussi publié avec Kevin Quinn, The Redeemed of the Lord Say So: a History of Queen's Theological College, 1912-1972 (Kingston, Queen's Theological College, 1980) et, tout récemment, Rawlyk, ed., Henry Alline. Selected Writings (New York, Paulist Press, 1987).

16 Par exemple, Study Sessions 1983. The Canadian Catholic Historical Association, 50 (1984). Voir aussi Terrence Murphy, «The Religious History of Atlantic Canada: the State of the Art», Acadiensis, 15,1 (1985): 152-174. 
les idées philosophiques qui ont bouleversé l'orthodoxie protestante victorienne dans les universités. Flanagan en histoire psychologique, Miller en mentalités politiques et McKillop en histoire des idées, ont élargi les frontières pour affronter des questions intéressant les chercheurs dans plusieurs domaines ${ }^{17}$. C'est dire que, depuis dix ans, l'historiographie s'identifie de moins en moins aux savants de Toronto et à l'étude des Églises institutionnelles dans leurs relations avec l'État.

Une science sociale de la religion s'impose comme troisième catégorie d'historiographie religieuse dans les multiples études publiées depuis dix ans. Au Canada anglais déchristianisé, l'analyse comparée des religions est un secteur en croissance. Dans les départements universitaires, les sociologues avaient fait table rase du passé. L'histoire se voulait ou bien un exercice littéraire à la Donald Creighton - mais le talent littéraire ne s'acquiert pas sur commande - ou un exercice presque rankéen, de plus en plus exigeant étant donné l'abondance de la documentation. Dans ce contexte, une science sociale se présentant avec ses hypothèses et ses problématiques, les historiens, fatigués et désireux de synthèse, ont accepté sans réticence les paradigmes sociologiques servant à exploiter - fut-ce brutalement - les données recueillies. Bref, une sorte d'école de sociologie phénoménologique a pris racine au Canada.

John Moir a signalé l'influence universitaire américaine au tournant du XXe siècle, par un échange d'étudiants en sciences bibliques entre McGill, Toronto, McMaster et Chicago. Pour sa part, Marlene Shore, dans son nouveau livre, The Science of Social Redemption, a montré comment, depuis Chicago, l'étude de la doctrine de l'évangile social a évolué vers les disciplines de travail social et de sociologie à l'Université McGill. Au fait, l'influence de Chicago ne se confine pas à l'histoire religieuse. Carl Berger a montré, de son côté, comment le Canadien Harold Innis, diplômé de Chicago, a transformé la pratique historique à l'Université de Toronto en introduisant l'étude de l'économie et de la société ${ }^{18}$.

Le Canada anglais compte maintenant une nouvelle génération d'historiens formés à Chicago. Keith Clifford en Colombie-Britannique et Tom Sinclair-Faulkner en Nouvelle-Écosse en sont les représentants

17 A. Brian McKillop, A Disciplined Intelligence: Critical Inquiry and Canadian Thought in the Victorian Era (Montréal, McGill-Queen's University Press, 1979). Thomas Flanagan, Louis "David» Riel. Prophet of the New World (Toronto, University of Toronto Press, 1979) et James R. Miller, Equal Rights: the Jesuits' Estates Act Controversy (Montréal, McGill-Queen's University Press, 1979). Â la suite de McKillop, Carl Berger du département d'histoire de l'Université de Toronto a écrit son petit Science, God, and Nature in Victorian Canada (Toronto, University of Toronto Press, 1983).

18 J. Moir, A History of Biblical Studies in Canada, 8, 17, 23-27, 71-76, etc. Marlene Shore, The Science of Social Redemption. McGill, the Chicago School, and the Origins of Social Research in Canada (Toronto, University of Toronto Press, 1987). Carl Berger, The Writing of Canadian History, 87-88. 
les plus accrédités ${ }^{19}$. Leurs professeurs de Chicago ont insisté sur l'utilisation des sciences sociales pour l'étude de la religion ${ }^{20}$. Avec l'aide de Robert Choquette, cet autre docteur de Chicago, les Canadiens anglais de l'école de Chicago se sont présentés devant un auditoire de francophones au colloque de la Société canadienne d'histoire de l'Église (surtout anglophone et protestante) en $1987^{21}$. Signe d'une volonté de rapprochement, cette dernière tiendra un colloque avec la Société canadienne d'histoire de l'Église catholique (francophone) à l'Université Laval en 1989. Alors que Choquette montre les quatre solitudes anglaise, française, protestante, catholique - dans l'historiographie religieuse au Canada, Keith Clifford met en relief les problèmes de l'histoire confessionnelle dans son livre The Resistance to Church Union in Canada. Par des analyses sociologiques, il apporte une nouvelle compréhension des anciens combats des presbytériens contre l'union ${ }^{22}$. Moir et Grant les avaient déjà décrits, mais Clifford explique la signification de l'évangile social pour les unionistes, et l'importance de l'infrastructure économique et sociale des régions comme le sud de l'Ontario pour les presbytériens. Il ajoute aussi que les femmes dans l'Église presbytérienne ne s'intéressaient pas toutes à un évangile social qui ne leur promettait qu'une libération incertaine ${ }^{23}$.

L'autre diplômé de Chicago, Tom Sinclair-Faulkner, n'a publié que des articles, mais son travail comme rédacteur en chef de Sciences religieuses nous amène à mettre en relief sa préférence pour une science sociale des religions comparées ${ }^{24}$. Il a convaincu la Société d'histoire

19 C. Thompson Sinclair-Faulkner, «For Christian Civilization»: the Churches and Canada's War Effort, 1939-1942, thèse de Ph.D., University of Chicago, 1975. Keith Markell, professeur à la faculté des sciences religieuses de l'Université McGill, a aussi reçu son doctorat de Chicago mais il a pris sa retraite en 1979. Avant sa mort en 1983, il a écrit History of the Presbyterian College, Montreal 1865-1986 (Montréal, 1987). Clifford a fait des études post-doctorales à Chicago.

${ }_{20}$ Robert Handy, The Protestant Quest for a Christian America 1830-1930 (Philadelphie, Fortress Press, 1967). Publié auparavant dans Church History, 22,1 (1952): 8-20. Voir aussi $A$ History of the Churches in the United States and Canada (New York, Oxford University Press, 1977 et 1979). Handy n'a pas enseigné à Chicago mais, baptiste, il y a reçu son diplôme. Joseph Ban et Paul Dekar, ed., In the Great Tradition. In Honor of Winthrop S. Hudson. Essays on Pluralism, Voluntarism, and Revivalism (Valley Forge, Pennsylvanie, Judson Press, 1982) est un autre exemple des relations entre Chicago et le Canada.

21 Il y avait Phyllis Airhart, The Eclipse of Revivalist Spirituality: the Transformation of Canadian Methodist Piety 1884-1925, thèse de Ph.D., University of Chicago, 1985). Professeure au Collège Emmanuel comme successeure de John Webster Grant, elle était secrétaire de la Société canadienne d'histoire de l'Église. Paul Dekar, Crossing Ritual Frontiers: Christianity and the Transformation of Bulu Society, 1892-1925, thèse de Ph.D., University of Chicago, 1978, est à l'Université McMaster et a organisé le colloque comme président de la Société.

22 Robert Choquette, «Les quatre solitudes en histoire religieuse canadienne», 23 avril 1987; N. Keith Clifford, «Denominational History: a Comparative Analysis of Its Problems and Prospects», 25 avril 1987; Joint Session of the American Society of Church History and the Canadian Society of Church History, Hamilton.

${ }^{23}$ N. K. Clifford, The Resistance to Church Union in Canada 1904-1939 (Vancouver, University of British Colombia Press, 1985).

24 Voir son «Editorial. Church History», Sciences religieuses, 16,3 (1987): 263-264. Aussi «Sacramental Suffering. Brother André's Spirituality», Canadian Society of Church History Papers 1982, i et 33, et aussi The Canadian Catholic Historical Association Study Sessions, 49 (1982): 
de l'Église d'exiger que toute personne désireuse de devenir membre s'abonne à la revue Sciences religieuses. Ainsi, bien qu'ayant peu publié, les adeptes de Chicago sont maintenant bien placés pour influer sur la direction de l'historiographie.

En conséquence, les historiens se divisent en deux camps. D'un côté, James Penton dans Apocalypse Delayed utilise la sociologie pour comprendre la théologie et l'évolution des Témoins de Jéhovah ${ }^{25}$. De l'autre, Ramsay Cook dans The Regenerators examine le processus de sécularisation à l'oeuvre à l'époque victorienne. Sans faire une étude sociologique, Cook trace le cheminement rapide de la sécularisation dans une société troublée par les nouvelles études bibliques, biologiques, communistes et cosmiques. Sa conclusion annonce que la théologie, en s'adaptant et en se tournant vers la terre plutôt que vers les cieux, a créé une société laïque. Conclusion logique. Cependant, l'omission par cet historien, autrefois de Toronto, de définir l'orthodoxie ou même la théologie protestante en dit long sur l'écart qui existe maintenant entre l'histoire et la théologie ${ }^{26}$. L'entente cordiale entre la théologie et l'histoire politique se détériore et se voit remplacée par les sciences sociales. L'avenir semble appartenir aux méthodes de l'histoire sociale de A. J. B. Johnston, qui a pu reconstruire la vie religieuse à Louisbourg de manière à étonner les historiens traditionnels ${ }^{27}$.

Si les praticiens de l'histoire politico-religieuse ont eu quelques réserves au sujet de Moon of Wintertime du théologien John Webster Grant, les adeptes d'une approche scientifique du fait religieux ont bien accueilli cet essai cherchant à comprendre les religions des indigènes. Son oeuvre entière a déjà inspiré un Festschrift sur les missions chrétiennes et d'autres ne tarderont pas $^{28}$. Mais les historiens du politique ne s'intéressent pas à l'entente que les théologiens chrétiens cherchent à fonder avec les théoriciens d'une science sociale de la religion. John Moir, par exemple, a démissionné de la Société d'histoire de l'Église

111-134. ««God's Flower of Hope»: the Religious Matrix of Québec's «indépendantisme»», William Westfall, Louis Rousseau, Fernand Harvey, John Simpson, eds., Religion/Culture. Etudes canadiennes comparées (Ottawa, Association des études canadiennes, 1985). Ses goûts pour la sociologie se révèlent dans «A Puckish Look at Hockey in Canada» qui s'inspire des sociologues Peter Berger et Thomas Luckmann ou «La religion se trouve en dehors des bornes des Églises et de la théologie», Peter Slater, ed., Religion and Culture in Canada/Religion et Culture au Canada (Toronto, Corporation canadienne des sciences religieuses, 1977), 383-405.

${ }_{25}$ Apocalypse Delayed. The Story of Jehovah's Witnesses (Toronto, University of Toronto Press, 1985). À comparer avec son Jehovah's Witnesses in Canada: Champions of Freedom of Speech and Worship (Toronto, Macmillan, 1976). Depuis qu'il s'est fait exclure des Témoins de Jehovah, Penton a adopté une approche sociologique comme Gregory Baum l'a fait dans Catholics and Canadian Socialism (Toronto, James Lorimer, 1980) en quittant la prêtrise.

${ }_{26}$ Ramsay Cook, The Regenerators: Social Criticism in Late Victorian English Canada (Toronto, University of Toronto Press, 1985). Pour une critique du livre de Cook, voir Randi Warne, «Post hoc ergo propter hoc: History, Theology and The Regenerators", The Canadian Society of Church History Papers 1986, 154-157.

27 A. J. B. Johnston, Religion in Life at Louisbourg, 1713-1758 (Montréal, McGill-Queen's University Press, 1985). Pour un survol, voir Carl Berger, The Writing of Canadian History, 267.

${ }_{28}$ J. Moir et C. T. McIntire, eds., Canadian Protestant and Catholic Missions, 1820 s1960s: Historical Essays in Honour of John Webster Grant (New York, Peter Lang, 1988). 
en 1988 quand la Société a imposé à ses membres la revue Sciences religieuses. D'autres historiens, tel Rawlyk, pourraient prendre sa place, mais ils pourraient aussi bien se voir remplacés par les sociologues dans les affections des théologiens ${ }^{29}$. À cet égard, il faut rappeler qu'en 1980, la Société d'histoire de l'Église s'est rassemblée à l'Université de Montréal. Une séance d'orientation avait pour titre: «L'histoire de l'Église au Canada: où aller?». Grant, Moir et Clifford animèrent la rencontre. Grant prétendit que l'histoire de l'Église continuerait dans sa voie - il avait raison. Clifford prophétisa la venue de l'histoire sociale sans frontière religieuse. John Moir a pensé qu'il était inutile de reproduire son propre texte dans les actes du colloque. Dix ans plus tard, nous constatons que chacun avait prédit son propre cheminement: Grant a continué, Clifford a frayé une piste et Moir est parti ${ }^{30}$. Un changement sur le plan des interrogations et des méthodes, le départ de professeurs et une préférence pour l'histoire analytique plutôt que pour l'histoire narrative ont tellement bouleversé l'histoire des relations entre les Églises et l'État que ce genre d'étude est en danger de disparition. Les nouveaux historiens réunissent universitaires et théologiens dans une interrogation de type sociologique. Au Canada, les adeptes de l'histoire sociale essaient de redéfinir l'histoire religieuse dans une perspective non confessionnelle. Ironiquement, les méthodes propres à la sociologie diachronique du fait religieux viennent de la sociologie, non de l'histoire. Or, comme la sociologie est une discipline en partie issue de la théologie chrétienne, nous soupçonnons que les questions futures seront celles des chrétiens - sécularisation, missions, orthodoxie, hétérodoxie, oecuménisme ${ }^{31}$.

Le fossé entre les solitudes décrites par Choquette semblait plus large au temps de Donald Creighton. Désormais, les historiens alignés sur «l'école» de Chicago, de même que Sinclair-Faulkner avec l'aventure de Sciences religieuses bilingue, annoncent la rencontre de ces solitudes. Peu sensible au nationalisme, cette nouvelle génération semble disposée à travailler avec les francophones, moins nationalistes eux aussi depuis 1980, séduits par l'histoire sociale et moins «confessionnels» depuis Vatican II. Les anglophones ont subi, à Chicago, une révolution méthodologique venant originellement d'Europe alors que les francophones se sont inspirés directement des courants européens.

29 La réception du livre de Hans Mol, Faith and Fragility: Religion and Identity in Canada (Burlington [Ont.], Trinity Press, 1985) est une indication de cette tendance.

${ }_{30}$ Clifford, Grant et Dekar, «Church History of Canada: Where From Here?», Canadian Society of Church History Papers 1980, 1-25.

31 Le livre de Brian J. Fraser, The Social Uplifters. Presbyterian Progressives and the Social Gospel in Canada, 1875-1915 (Waterloo [Ont.], Corporation canadienne des sciences religieuses, Wilfrid Laurier University Press, 1988) est classé d'abord comme sociologie chrétienne et en second lieu comme oeuvre d'histoire. Il n'est pas sans intérêt de savoir que ce livre est un Sciences religieuses supplement. 
Les méthodes sociologiques du troisième courant semblent devoir être le moyen d'éviter le nationalisme et le particularisme confessionnel des deux premiers. Ce troisième courant surgit de la rencontre des théologiens et des sociologues. En fixant le regard sur l'horizon lointain, on croit apercevoir le rapprochement des lignes parallèles d'historiographie religieuse canadienne - anglaise et française, protestante et catholique. Serait-ce encore une illusion d'optique? 